\title{
Cambio en la esperanza de vida según tres grandes grupos de causas de muerte en Medellín, Colombia, de 1989-1991 a 1994-1996
}

\author{
Hugo Grisales Romero, ${ }^{1}$ Gloria Stella Estrada Bedoya, ${ }^{1}$ \\ Mónica Eliana Aristizábal Velásquez y Maryluz Posada Marín ${ }^{1}$
}

RESUMEN Objetivos. Examinar los cambios en la esperanza de vida en Medellín, Colombia, entre los trienios de 1989-1991 y 1994-1996, en relación con los cuatro grupos de causas de muerte utilizados convencionalmente en los estudios sobre la carga de morbilidad: grupo 1, enfermedades transmisibles, perinatales, maternas y nutricionales; grupo 2, enfermedades no transmisibles; grupo 3, traumatismos; grupo 4, causas mal definidas. Estas últimas se excluyeron del análisis debido a su ambigüedad.

Métodos. Para hacer los cálculos se utilizó el método descrito por J. H. Pollard en 1986, con ayuda del programa Microsoft Excel, usando las fórmulas requeridas para su aplicación. Las operaciones fueron verificadas con el programa EPIDAT (versión 3.0 no oficial).

Resultados. Entre el primer y segundo trienio (1994-1996 y 1989-1991, respectivamente) se observó una ganancia total de 1,93 años en la esperanza de vida al nacer, la cual subió de 62,13 a 64,06 años. Esta ganancia fue mayor en los hombres que en las mujeres (2,42 y 1,09 años, respectivamente). Al aplicar el método de Pollard, se advirtió una ganancia en la esperanza de vida general de 2,03 años para el total de la población. La ganancia en el sexo femenino se concentró en las edades extremas (niñas de 1 a 4 años y mujeres mayores de 54), y en los hombres en las edades intermedias (entre los 25 y 44 años). Se destaca, además, que en ambos sexos la mayor pérdida porcentual en la esperanza de vida se presentó en el grupo de 15 a 19 años (23\% en los hombres y 4\% en las mujeres, aproximadamente).

El grupo 1 de causas de defunción se asoció con una pérdida de la esperanza de vida en los hombres $(0,04$ años), mientras que en el grupo 2 se observó en ambos sexos un aumento de dicha esperanza $(0,60$ años para los hombres y 0,55 años para las mujeres). Se destaca que la mayor ganancia masculina en la esperanza de vida se relacionó con una reducción de la mortalidad por traumatismos (1,98 años). Conclusiones. Si la esperanza de vida al nacer observada en Medellín al final del período de estudio se compara con la esperanza de vida en toda Colombia en 1995, que era de 70 años, se advierte que la cifra en Medellín sigue siendo inferior a la de todo el país, pese a que ha aumentado paulatinamente en el transcurso de los años. Este fenómeno refleja la transición epidemiológica observada en la ciudad, que se caracteriza por un aumento de la mortalidad por enfermedades degenerativas, trastornos cardiovasculares, enfermedades respiratorias crónicas y diabetes, así como por enfermedades cuya causa se debe a actividad humana. No obstante, en Medellín, como en toda Colombia, esta transición epidemiológica ha sido lenta y tenaz y se ha reflejado primariamente en el sexo femenino. La poca participación que los hombres han tenido en ella se debe a su gran mortalidad a causa de traumatismos intencionales. Pese a que en Medellín ha disminuido la mortalidad por traumatismos, particularmente en la población masculina joven, cabe reexaminar la eficacia de las intervenciones efectuadas en los últimos años con el fin de fomentar la convivencia pacífica y la tolerancia en la comunidad.

Palabras clave Esperanza de vida, método de Pollard, causas de defunción.

1 Universidad de Antioquia, Facultad Nacional de Salud Pública, Medellín, Colombia. Dirección postal: Calle 62 No. 52-19 (oficina 208), Medellín, Colombia. Correo electrónico: hgrisales@guajiros. udea.edu.co
En los diferentes estudios de mortalidad que se han hecho en la ciudad de Medellín se han utilizado distintos métodos e indicadores, entre ellos las tasas de mortalidad, los años de vida potencial perdidos (APVP), los años de 
vida saludable perdidos (AVSP) y la esperanza de vida al nacer. Mediante todos ellos se ha logrado una aproximación al perfil de salud de la comunidad $(1,2)$. Sin embargo, es necesario complementar esos estudios para dar respuesta a interrogantes que surgieron a raíz del análisis de la situación de salud de la población de Medellín en el período de 1987 a 1996, durante el cual en la ciudad hubo diversos fenómenos de salud que influyeron en su transición epidemiológica $(3,4)$, principalmente un aumento de las enfermedades degenerativas y cardiovasculares, cáncer, diabetes, enfermedades respiratorias crónicas y enfermedades digestivas (5). Estas interrogantes se orientan a lograr un conocimiento más profundo del cambio en la esperanza de vida de los habitantes de la ciudad, de la manera en que la mortalidad por distintas causas ha contribuido a ese cambio, y de las diferencias de mortalidad entre los sexos y grupos de edad (6).

A la luz de los antecedentes históricos de las dos últimas décadas en Medellín (7) y de los resultados de estudios previos (8), reviste particular interés saber si ha cambiado la esperanza de vida entre los años 19891991, cuando la violencia se manifestó con mayor intensidad en la ciudad, y 1994-1996 (por ser estos los años más recientes para los cuales se cuenta con información de mortalidad por causas, codificadas según la Clasificación internacional de enfermedades, novena revisión).

El presente estudio tiene por objetivo determinar el aporte de las distintas causas de muerte al cambio en la esperanza de vida usando una metodología que propuso J. H. Pollard en 1986. La metodología, que es relativamente novedosa, permite determinar en qué medida estas causas, desglosadas por sexo y edad, contribuyeron a la ganancia o pérdida de esperanza de vida en el período definido (6). Se espera que la información arrojada por el presente estudio oriente a los organismos pertinentes a la hora de formular medidas encaminadas a reducir el riesgo de morir y ayude a aumentar la esperanza de vida de la población de Medellín $(9,10)$.

\section{MATERIALES Y MÉTODOS}

Aunque los indicadores de ganancia o pérdida en la esperanza de vida se obtuvieron individualmente para cada una de las causas recogidas en la Clasificación internacional de enfermedades, novena revisión, estas se agruparon en los siguientes cuatro grandes grupos: en el primero se incluyeron las enfermedades transmisibles, perinatales, maternas y nutricionales por tratarse de las afecciones que más prevalecen en las primeras etapas de la transición epidemiológica. En los grupos segundo, tercero y cuarto se ubicaron las enfermedades no transmisibles, los traumatismos y las causas mal definidas, respectivamente. Se eligió esta clasificación por ser la utilizada en las investigaciones acerca de los AVSP por una población como resultado de las distintas enfermedades $(11,12)$.

Para determinar el efecto que tuvo la mortalidad por cada grupo de causas de defunción sobre la ganancia o pérdida de la esperanza de vida en años, se utilizó el método de cálculo de Pollard con ayuda del programa Microsoft Excel usando un formato basado en las fórmulas requeridas para su aplicación. También se recurrió al programa EPIDAT (versión 3.0 no oficial), para verificar estas operaciones.

El cambio en la esperanza de vida entre dos momentos, llamados 1 y 2 , se puede descomponer del modo siguiente (13):

$$
\begin{aligned}
e_{0}^{2} \pm e_{0}^{1} \cong & \sum_{j} \sum_{x} n w_{x+n / 2}\left({ }_{n} m_{x}^{1, j} \pm_{n} m_{x}^{2, j}\right)= \\
= & \sum_{j}\left[w_{0}\left({ }_{1} m_{0}^{1, j} \pm_{1} m_{0}^{2, j}\right)+\right. \\
& +4 w_{3}\left({ }_{4} m_{1}^{1, j} \pm_{4} m_{1}^{2, j}\right)+ \\
& +5 w_{7,5}\left({ }_{5} m_{5}^{1, j} \pm_{5} m_{5}^{2, j}\right)+ \\
& \quad \cdots \\
& +5 w_{77.5}\left({ }_{5} m_{75}^{1, j} \pm_{5} m_{75}^{2, j}\right)+ \\
& \left.+\left(m_{80}^{1, j} \pm m_{80}^{2, j}\right)\right]
\end{aligned}
$$

En donde:

- j representa la causa de defunción. Si se estudia cuánto contribuye una sola causa de muerte al cambio en la esperanza de vida, entonces las defunciones por esa causa se comparan con el resto de las defunciones.
Así, la suma en $j$ se descompondría en dos sumandos: uno para la causa en consideración y otro para el resto de las causas.

- $x$ representa la edad inicial del intervalo de edad y $n$ es la amplitud del intervalo. Si los grupos de edad se dividen por quinquenios, exceptuando las edades de 0 a 1,1 a 4 y más de 80 años, entonces si $x=0$, $\mathrm{n}=1$; si $x=1, \mathrm{n}=4$; si $x=5,10,15$, $20 \ldots 75, n=5$.

- ${ }_{n} m_{x}^{i, j}$ es la tasa de mortalidad entre las edades $x$ y $(x+n)$ por la causa $j$ en la tabla $i,(i=1,2)$,

- $w_{x}=0,5\left({ }_{x} p_{0}^{1} e_{x}^{2}+{ }_{x} p_{0}^{2} e_{x}^{1}\right)$, representa una función de ponderación de la edad.

- ${ }_{x} p_{\mathrm{o}}^{i}$ es la probabilidad de sobrevivir hasta la edad $x$ en la tabla $i$,

- $e_{x}^{i}$ es la esperanza de vida a la edad $x$ en la tabla $i$.

Para el último grupo abierto se toma $n w_{x+n / 2}=1$.

Los pesos $w_{x}$ se calculan tomando las probabilidades de supervivencia en el punto medio de cada intervalo de edad. Las probabilidades de sobrevivir se obtienen a partir de la serie de supervivientes, y la probabilidad de supervivencia en el punto medio del intervalo de edad $(x, x+n)$ se calcula como la media aritmética de las probabilidades en los extremos, es decir:

$$
{ }_{x+n / 2} p_{0}^{i}=\frac{{ }_{x} p_{0}^{i}+{ }_{x+n} p_{0}^{i}}{2}=\frac{l_{x}^{i}+l_{x+n}^{i}}{2 l_{0}^{i}}
$$

lx son las personas que han sobrevivido hasta la edad $x$. Generalmente el valor de $l_{0}$ en las tablas de vida es igual a 100000 .

El insumo básico para aplicar el método de Pollard consiste en las funciones de las tablas de vida, que fueron construidas con el paquete PANDEM v. 1.0 a partir de una fuente secundaria conformada por la base de datos de los certificados de defunción registrados por el Departamento Administrativo Nacional de Estadística (DANE) para las muertes ocurridas en Medellín durante los trienios de 1989-1991 y 19941996 (14). Los datos sobre la población de Medellín por grupos de edad y sexo para los mismos años se tomaron de 
estudios anteriores realizados en la ciudad (15) y de fuentes de información secundarias, tales como el Anuario estadístico metropolitano, el Boletín epidemiológico de Antioquia e informes de proyecciones de población de la oficina de planeación de la ciudad de Medellín. con el fin de calcular las tasas de mortalidad por edad y sexo, pero la información más importante para las estimaciones necesarias la proporcionó el Censo Nacional de Población y Vivienda de 1993.

Para facilitar su manejo, las causas de muerte codificadas con tres dígitos, según la novena revisión de la Clasificación internacional de enfermedades, se dividieron en cuatro grandes grupos. Se seleccionaron estos grupos por ser los que se usan convencionalmente en los estudios que miden la carga de morbilidad (16-18). El objeto fue resumir fácilmente la información obtenida y atenuar algunos errores que pueden presentarse en los registros de defunción $(19,20)$, utilizando el paquete EPIINFO v. 6.04. Se excluyeron las ganancias o pérdidas de la esperanza de vida correspondientes a las defunciones por causas "mal definidas", las cuales representaron $0,6 \%$ del total de 155745 defunciones registradas en el período de estudio. El aporte al cambio en la esperanza de vida de cada uno de los otros tres grupos de causas de mortalidad incluidas en el análisis se ilustra por medio de figuras que muestran su contribución positiva (ganancia) o negativa (pérdida), por sexo y grupo de edad.

\section{RESULTADOS}

Durante el decenio de 1987 a 1996, las tasas de mortalidad general en Medellín fluctuaron entre 7,61 y 9,83 defunciones por 1000 habitantes, siendo el riesgo de morir aproximadamente el doble en el sexo masculino que en el femenino (15).

Al dividir todas las causas de muerte en los tres grandes grupos utilizados por convención en los estudios de la carga de morbilidad (18), se encontró que la mortalidad por las enfermedades transmisibles, perinatales, mater- nas y nutricionales (grupo 1) se mantuvo estable durante todo el decenio, aunque en los últimos dos años del período mostró una tendencia ascendente que fue similar para ambos sexos. La mortalidad por enfermedades no transmisibles (grupo 2) aumentó en 1993, y a fines del decenio de 1987-1996 mostró fluctuaciones similares en hombres y mujeres. Las defunciones por traumatismos (grupo 3) mostraron distintas tendencias en hombres y mujeres. Los primeros tuvieron altas tasas de mortalidad, especialmente entre 1989 y 1993, que posteriormente disminuyeron. En las mujeres, las tasas de mortalidad fueron mucho menores que en los hombres y se mantuvieron estables durante todo el decenio.

Tomando como referencia las tablas de vida construidas para cada uno de los dos trienios que delimitaron el período de estudio, la población de Mede- llín obtuvo una ganancia de 1,93 años en la esperanza de vida al nacer, gracias a un ascenso de 62,13 a 64,06 años durante los cinco años comprendidos en el intervalo entre los dos trienios. Dicha ganancia fue mayor en los hombres (2,42 años) que en las mujeres (1,09 años); en el segundo trienio, la esperanza de vida al nacer de hombres y mujeres fue de 56,47 y 71,88 años, respectivamente.

La mayor ganancia en esperanza de vida que mostraron las mujeres se presentó en los extremos de edad (mujeres de 1 a 4 años y mayores de 54) de acuerdo con los resultados obtenidos aplicando el método de Pollard. Lo contrario ocurrió en los hombres, cuya ganancia en esperanza de vida fue mayor en los grupos de edad intermedios (de 25 a 44 años) (figura 1). Asimismo, la esperanza de vida se vio afectada desfavorablemente por la alta mortalidad femenina en el grupo de

FIGURA 1. Ganancia (+) o pérdida (-) porcentual de la esperanza de vida, por grupo de edad y sexo, calculada mediante el método de Pollard. Medellín, 1989-1991 y 1994-1996

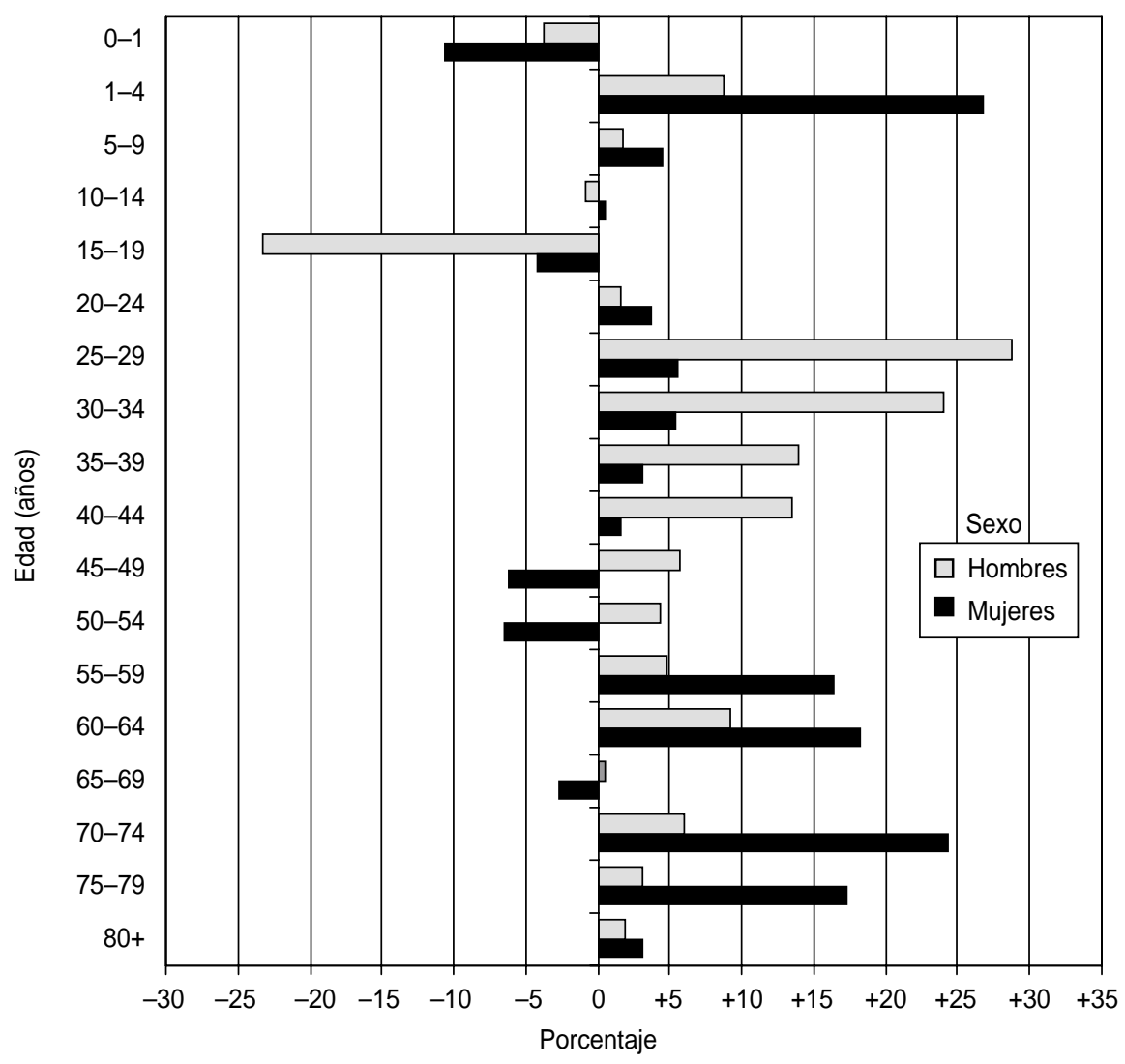


FIGURA 2. Años de esperanza de vida ganados (+) o perdidos (-), según tres grandes grupos de causas de muerte, por sexo, calculados mediante el método de Pollard. Medellín, $1989-1991$ y $1994-1996$

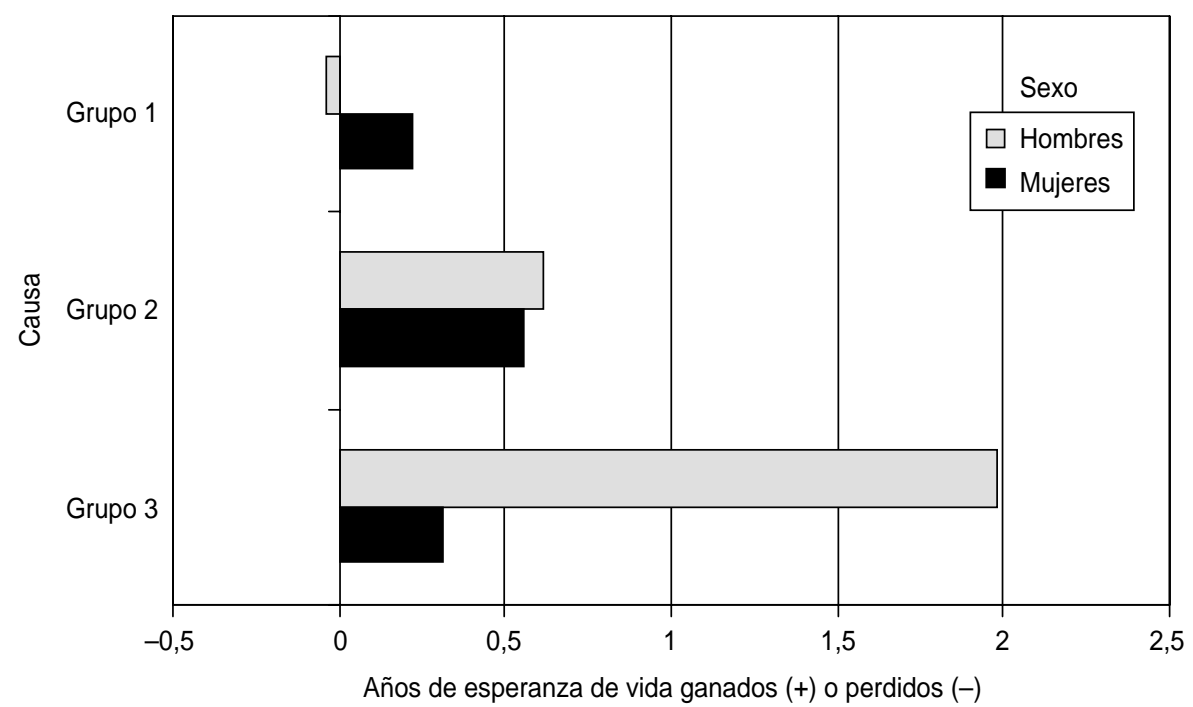

Grupo 1: Enfermedades transmisibles, perinatales, maternas y nutricionales. Grupo 2: Enfermedades no transmisibles.

Grupo 3: Traumatismos.

CUADRO 1. Años de esperanza de vida ganados (+) o perdidos (-), según tres grandes grupos de causas de muerte, por sexo, calculados mediante el método de Pollard. Medellín, 1989-1991 y 1994-1996

\begin{tabular}{lccc}
\hline \multirow{2}{*}{ Grupo } & \multicolumn{2}{c}{ Sexo } & \\
\cline { 2 - 3 } & Hombres & Mujeres & Total \\
\hline $\begin{array}{l}\text { Enfermedades transmisibles, } \\
\text { perinatales, maternas y nutricionales }\end{array}$ & & & \\
$\quad$ Total & $-0,0415$ & $+0,2225$ & $+0,0739$ \\
$\%$ & $-1,61$ & $+19,89$ & $+3,64$ \\
$\begin{array}{l}\text { Enfermedades no transmisibles } \\
\quad \text { Total }\end{array}$ & $+0,6181$ & $+0,5549$ & $+0,5977$ \\
$\quad \%$ & $+23,91$ & $+49,59$ & $+29,45$ \\
$\begin{array}{l}\text { Traumatismos } \\
\quad \text { Total }\end{array}$ & $+1,9800$ & $+0,3108$ & $+1,3278$ \\
$\quad \%$ & $+76,59$ & $+27,77$ & $+65,41$ \\
$\begin{array}{l}\text { Causas mal definidas } \\
\quad \text { Total }\end{array}$ & $+0,0284$ & $+0,0307$ & $+0,0306$ \\
$\%$ & $+1,1$ & $+2,74$ & $+1,50$ \\
\hline
\end{tabular}

${ }^{a}$ Las causas mal definidas no fueron incluidas en el análisis de los datos. No obstante, los valores correspondientes se colocaron en el cuadro para que se pueda apreciar su contribución relativa al total.

menores de 1 año y en los grupos de 15-19 años, 45-54 años y 65-69 años. También se vio menoscabada por la mortalidad masculina en menores de un año y en varones de 10 a 14 años y de 15 a 19. Este último grupo presentó la más alta pérdida porcentual en esperanza de vida $(23,29 \%)$ (figura 1 ).

La esperanza de vida mostró el mayor aumento en conexión con la mortalidad por traumatismos (grupo 3), siendo más alta la ganancia en los hombres que en las mujeres. En cambio, la esperanza de vida en los hombres bajó 0,04 años en relación con la mortalidad por causas de defunción del grupo 1, destacándose en este las enfermedades infecciosas y parasitarias, las infecciones respiratorias y las enfermedades nutricionales. En el caso de las mujeres, el mayor aumento en la esperanza de vida (0,55 años) se concentró en el grupo 2 de causas de mortalidad, conformado por las enfermedades no transmisibles (figura 2).

\section{Defunciones por distintas causas}

Grupo 1: Enfermedades transmisibles, perinatales, maternas y nutricionales. La contribución total de este grupo de causas a la ganancia general en la esperanza de vida calculada mediante el método de Pollard fue de 0,07 años, cifra que equivale a $3,64 \%$ del total de años de esperanza de vida ganados en todo el período estudiado (cuadro 1). Se observó una ganancia de 0,22 años en la esperanza de vida de las mujeres. Sin embargo, en 8 de los 18 grupos de edad se advirtieron pérdidas. En el caso de los hombres, se observó una pérdida en la esperanza de vida en general de 0,04 años, la cual se concentró en los grupos de 10 a 59 años de edad (figura 3).

Grupo 2: Enfermedades no transmisibles. La contribución total de este grupo de causas a la ganancia de esperanza de vida fue de 0,60 años, o $29,45 \%$ del aumento general en ambos sexos. Esta causa aportó el mayor número de años a la ganancia en la esperanza de vida de las mujeres. La reducción de la mortalidad por enfermedades no transmisibles redundó en una ganancia en esperanza de vida para ambos sexos, según el método de Pollard, habiendo ganado más años los hombres que las mujeres $(0,62 \mathrm{y}$ 0,55 años, respectivamente) (cuadro 1). No obstante, se encontró una pérdida en la esperanza de vida en hombres de 10 a 19 años de edad, y en mujeres en varios grupos de edad, especialmente 
FIGURA 3. Años de esperanza de vida ganados (+) o perdidos (-), según tres grandes grupos de causas de muerte, por edad y sexo, calculados mediante el método de Pollard. Medellín, 1989-1991 y 1994-1996

Grupo 1: Enfermedades transmisibles, perinatales, maternas y nutricionales

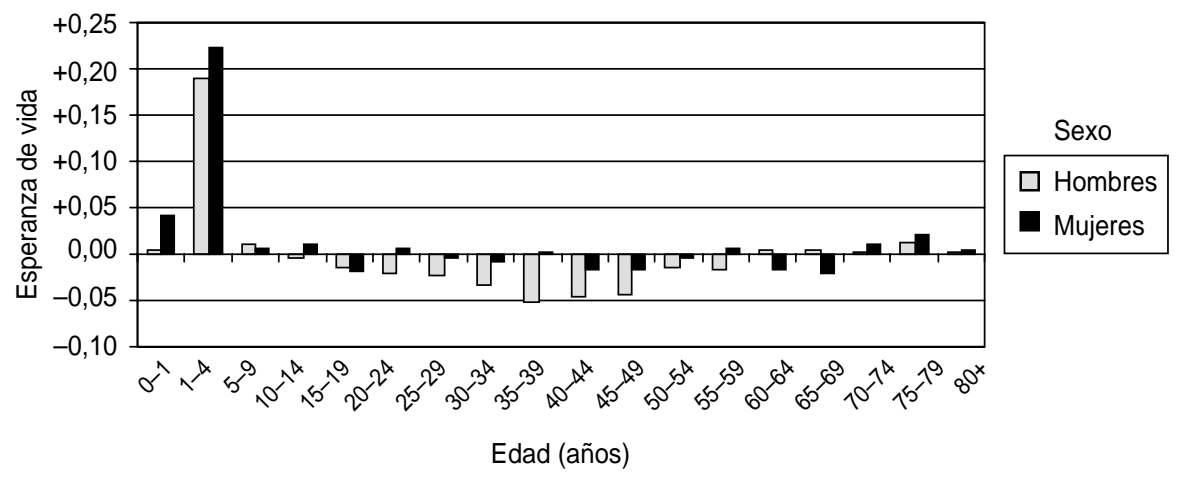

Grupo 2: Enfermedades no transmisbles

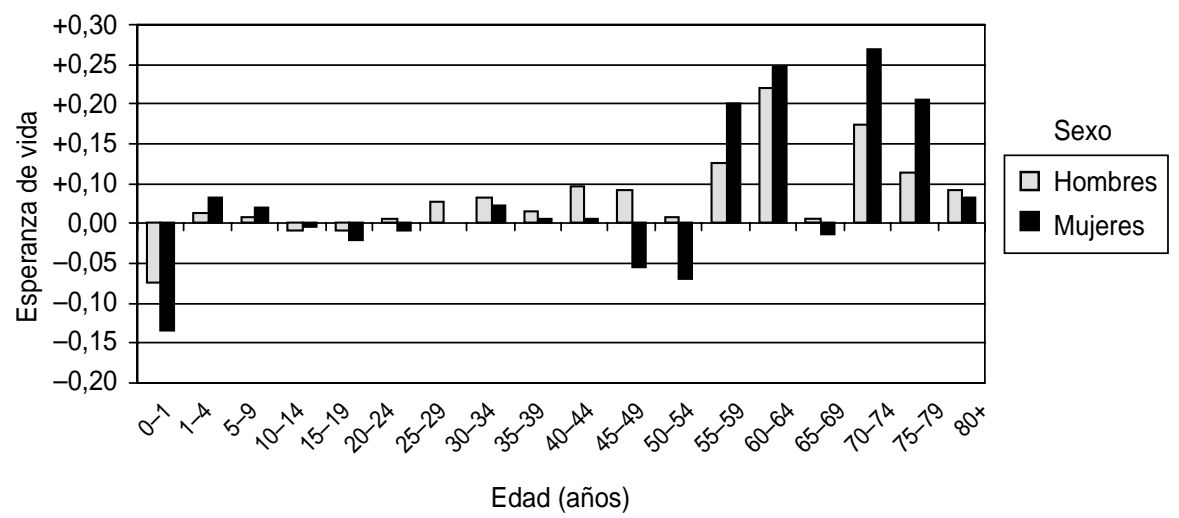

Grupo 3: Traumatismos

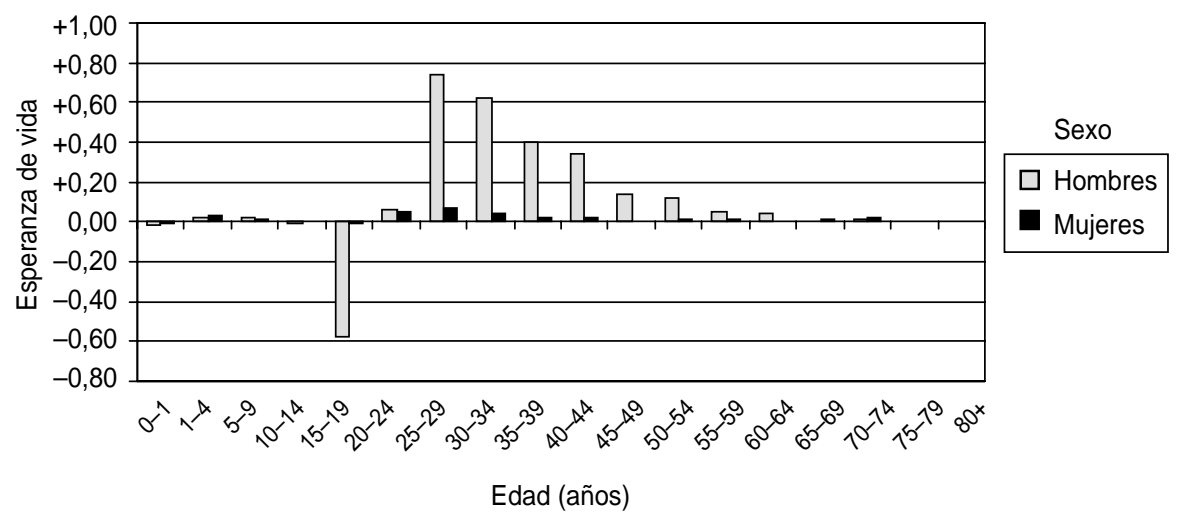

entre los 10 y 29 años. En ambos sexos se observó una pérdida en niños menores de 1 año (figura 3).

Grupo 3: Traumatismos. Entre los dos trienios, la contribución del grupo de los dos períodos estudiados. ${ }^{2}$ El aumento fue mayor en los hombres que en las mujeres, con 1,98 años y 0,31 años ganados, respectivamente (cuadro 1). Hubo una pérdida en la esperanza de vida en varones de 10 a 19 años y en los menores de un año. Sin embargo, el grupo de los traumatismos fue el que más contribuyó a aumentar la esperanza de vida de los hombres entre los dos trienios (figura 3).

\section{DISCUSIÓN}

En Medellín se produjo, entre los trienios de 1989-1991 y 1994-1996, una ganancia total de 1,93 años en la esperanza de vida al nacer, la cual subió de 62,13 a 64,06 años, y una contribución total de 2,03 años de esperanza de vida general, calculada por el método de Pollard. Si se compara este aumento con la esperanza de vida al nacer en toda Colombia en 1995, que era de 70 años (21), se advierte que la esperanza de vida en Medellín sigue siendo inferior, aunque refleja un ascenso paulatino en el transcurso de los años. Este fenómeno es consecuente con la transición epidemiológica observada en la ciudad, caracterizada por la mayor incidencia de enfermedades degenerativas, trastornos cardiovasculares, enfermedades respiratorias crónicas y diabetes, así como de las enfermedades producidas por el hombre $(3,6)$. Esta transición epidemiológica ha sido lenta en Colombia $(18,27)$, en parte debido a que las lesiones intencionales han ejercido un gran peso en la mortalidad de la población $(22,23)$. Además, está polarizada porque la pérdida en esperanza de vida es mayor en los estratos marginados social y económicamente que en el resto

2 Hay una diferencia entre el cambio en la esperanza de vida al nacer y la ganancia o pérdida en la esperanza de vida calculada por el método de Pollard, ya que esta última se basa en todos los grupos de edad, mientras que la esperanza de vida solo contempla el grupo de edad de los menores de un año. En este documento se notifica una ganancia global de la esperanza de vida al nacer de 1,93 años, según el modelo de Coale-Demeny (tablas para el mundo occidental). Sin embargo, cabe advertir que el aumento en la esperanza de vida (2,03 años), teniendo en cuenta los aportes de todos los grupos de edad por sexo y causa de muerte, fue mayor que el de la esperanza de vida al nacer. 
de la población. Estos resultados, sin embargo, no reflejan una situación de tipo determinista; más bien, representan una síntesis histórica del proceso salud-enfermedad-muerte, que encierra, entre otras cosas, las contribuciones de las condiciones de vida, la política social del estado y la prestación de servicios de salud a la comunidad (7).

De un trienio a otro, la diferencia en la esperanza de vida al nacer de hombres y mujeres se redujo en Medellín. Esto obedeció a que la esperanza de vida al nacer de los hombres aumentó en mayor medida que la de las mujeres. No obstante, en el segundo trienio el sexo masculino presentó una esperanza de vida (56,47 años) muy inferior a la del país en general (70 años en 1995) y a la del sexo femenino en particular (71,88 años). Obsérvese que en el segundo trienio la diferencia en la esperanza de vida al nacer de hombres y mujeres era mayor de 15 años, en tanto que en un estudio sobre la mortalidad evitable que se realizó en 19761992 en Galicia, España (13), se obtuvo una esperanza de vida general de 77,5 en hombres y de 81,5 en mujeres, lo cual representa una diferencia de ocho años entre los sexos. La mayor magnitud de esta diferencia en Medellín podría deberse a la violencia que ha asolado a la ciudad en los últimos años, la cual ha generado un exceso de mortalidad en la población masculina. No obstante, el aumento de la esperanza de vida de los hombres de un trienio a otro es indicio de que la tendencia empieza a modificarse $(15,24)$.

Al examinar el aporte de cada grupo de edad al cambio en la esperanza de vida según los resultados obtenidos al aplicarse el método de Pollard, resulta evidente que los menores de un año han aportado una pérdida, probablemente como consecuencia de un recrudecimiento de la mortalidad por enfermedades propias de la primera infancia. Esto suscita dudas acerca de las estrategias encaminadas a reducir la mortalidad infantil en Medellín. Sin embargo, en casi todo el resto de las edades hubo ganancias importantes. En los hombres, por ejemplo, se destacan los aportes positivos a la esperanza de vida en las edades jóvenes, que se consideran las de mayor riesgo de morir; y entre las mujeres las ganancias se produjeron principalmente en las edades avanzadas, cuyo riesgo de morir obviamente ha disminuido (19).

En el grupo de las lesiones se destaca que en las edades entre 20 y 44 años se presentó la mayor ganancia de esperanza de vida a pesar de que estudios anteriores mostraron que el mayor número de años de vida potencial y más de la mitad de los años de vida saludable perdidos por la población de Medellín entre 1994 y 1996 se debió a este grupo de causas, siendo dicha pérdida mucho más grande en los hombres que en las mujeres $(5,25)$. Sin embargo, en el grupo de edad de 15 a 19 años hubo una pérdida en la esperanza de vida en ambos sexos, aunque fue superior en los hombres. Estos resultados se asemejan a los de un estudio de mortalidad entre la gente joven y su impacto en la esperanza de vida, el cual se llevó a cabo en Andalucía, España, 1980-1992. En dicho estudio, los hombres tuvieron un efecto adverso en la esperanza de vida como consecuencia de la alta mortalidad por accidentes de tránsito, suicidios y otros traumatismos (grupo 3) en varones de 15 a 40 años de edad (26). En un estudio sobre las causas de la disminución de la esperanza de vida en Rusia (1990-1994), se encontró que los traumatismos contribuyeron notablemente a esa pérdida, pero principalmente en la edad adulta y los extremos de edad, resultados que no coinciden con los del presente estudio (27).

A pesar de que en Medellín ha disminuido la mortalidad por traumatismos, particularmente en la población masculina joven, sigue habiendo dudas acerca de la eficacia de los programas de intervención que se han implementado en los últimos años con el fin de fomentar en la comunidad la convivencia pacífica y la mutua tolerancia (15). En este grupo de causas es importante resaltar el aporte positivo a la esperanza de vida de la disminución de la mortalidad por ataque con armas de fuego y materiales explosivos, la cual contribuyó 1,24 años a los 2,03 años ganados en total, a pesar de que en ambos trienios esta causa se asoció con el mayor número de defunciones. El sub- grupo compuesto por las defunciones por ataque con instrumentos cortantes y punzantes, que ocuparon el segundo lugar en frecuencia en ambos trienios, también tuvo un aporte favorable a la esperanza de vida. Aunque las muertes por estas dos causas han disminuido de un trienio a otro, debido probablemente a la lucha frontal que libra el estado colombiano contra el narcotráfico y sus diferentes manifestaciones, específicamente en la ciudad de Medellín, han surgido otras causas de violencia derivadas de la situación política y social del país. Entre ellas se encuentran la proliferación de grupos armados ilegales, el constante clima de intolerancia avivado por las denominadas bandas que pululan en los barrios de la periferia y el desplazamiento del campesino a la ciudad, factor que incrementa la posibilidad de que se produzcan actos de violencia. Esta situación de alguna manera contribuye a que en la ciudad de Medellín las defunciones por causas asociadas con la violencia ocupen un lugar destacado.

También merece especial atención el grupo de las enfermedades no transmisibles, que habiendo aportado más de medio año a la ganancia en la esperanza de vida en Medellín, contribuyó con $26,1 \%$ de los años de vida potencial perdidos (20) y 31,8\% de los años de vida saludable perdidos (25), sin grandes diferencias por sexo, en el período de 1994-1996. Estos hallazgos son coherentes con los resultados obtenidos en un estudio realizado en Japón, según el cual las enfermedades cerebrovasculares, que hacían el mayor aporte a la mortalidad en ese país, disminuyeron significativamente entre 1972 y 1982, con lo que aumentó la esperanza de vida de las personas mayores de 55 años (28). Dentro de este grupo de causas, la diabetes mellitus, el infarto agudo de miocardio y la obstrucción crónica de las vías respiratorias contribuyeron a la pérdida de la esperanza de vida, especialmente en las mujeres. En el caso de la diabetes mellitus, surgen interrogantes en torno a la forma de certificar la muerte, ya que la presencia en Medellín de un programa de diabetes puede hacer que los médicos sean más propensos a indi- 
carla entre las causas de defunción. También es posible que haya empeorado la atención de los pacientes diabéticos y que, en consecuencia, la defunción sea más frecuente. No es común que una enfermedad crónica que está estabilizada en la población, como la diabetes mellitus, menoscabe la esperanza de vida. El infarto agudo del miocardio se asoció con pérdidas y ganancias en la esperanza de vida en menores de 5 años, lo cual es indicio de que la calidad de los datos de defunción no es fiable. Incluso el Instituto Nacional de Salud y el Ministerio de Salud de Colombia aconsejaron en 1998 utilizar la historia clínica del paciente como instrumento de recolección de datos para futuros estudios. Aunque esta medida permite tener cierta seguridad al usar el diagnóstico de infarto agudo del miocardio con fines de investigación, tiene la desventaja de que solo cubre la mortalidad institucional.

El grupo de las enfermedades no transmisibles no parece haber tenido mayor efecto sobre la esperanza de vida. No obstante, es necesario poner en marcha estrategias y programas orientados a promover estilos de vida saludables, de tal manera que la comunidad se eduque en materia de salud a manera de prevenir estas enfermedades $(10,29)$. Este grupo de causas, que se consideran evitables y propias del proceso de modernización y desarrollo que va avanzando cada vez más en la ciudad de Medellín, requiere, además, una atención adecuada basada en un diagnóstico precoz y un tratamiento oportuno (7).

El grupo de las enfermedades transmisibles, perinatales, maternas y nutricionales, que contribuyó en 3,64\% al aumento de la esperanza de vida, no tuvo un aporte muy grande si se tiene en cuenta que una característica de la transición demográfica es el desplazamiento de las causas de muerte hacia las enfermedades no transmisibles (3). A pesar de que hubo una ganancia en la esperanza de vida en general, se observó una pérdida en los grupos de edad de 15 a 69 años, principalmente en el sexo masculino. Las causas específicas de mortalidad que explican esta pérdida, que son las enfermedades infecciosas y parasitarias, las infecciones respiratorias y las enfermedades nutricionales, no ocupan un lugar muy importante en el perfil epidemiológico de la ciudad de Medellín. El grupo de edad de 0 a 4 años obtuvo una ganancia en la esperanza de vida $(0,21$ años) que fue más pronunciada en el grupo de 1 a 4 años de edad. En un estudio realizado por Londoño et al. (5), la mortalidad perinatal durante los primeros días de vida ocasionó el mayor número de AVPP. Es posible que esta pérdida se encuentre estrechamente asociada a las malas condiciones socioeconómicas en que viven amplios sectores de la población (30).

Agradecimientos. Los autores expresan su agradecimiento a Soly Santiago de la Xunta de Galicia, España; a Enrique Vásquez de la Dirección Xeral de Saúde Pública de Galicia, España, responsable del formato de Excel y de la verificación del análisis en EPIDAT (correo electrónico: dxsp19@ jet.es); a María Eugenia Mazuera del Hierro, Jefa del Centro de Investigaciones de la Facultad Nacional de Salud Pública de la Universidad de Antioquia; a Sara Yaneth Fernández, Héctor Byron Agudelo García y Alfonso Helí Marín Echeverri del grupo de investigación de Demografía y Salud, al Departamento Administrativo Nacional de Estadística (DANE) y al Comité para el Desarrollo de la Investigación (CODI).

\section{REFERENCIAS}

1. Giraldo P, Molina A. Tasas de mortalidad, años de vida potenciales perdidos y años de vida saludable perdidos como indicadores epidemiológicos para la identificación de prioridades en salud: un análisis comparativo, Colombia 1994. Bol Epidemiol Antioquia 1996;21(1):79-93.

2. Avalos O. Orientaciones metodológicas generales para el estudio de los distintos componentes de la mortalidad de la población. Rev Cubana Admin Salud 1987;13(2):273-279.

3. Frenk J, Lozano R, Bobadilla J. La transición epidemiológica en América Latina. Rev Notas Población 1994;22(60):79-99.

4. Pabón A. La mortalidad en Colombia 19531991: frecuencias por edad y sexo. Causas de defunción. Santa Fe de Bogotá: Instituto Nacional de Salud; 1993.

5. Londoño J, Grisales H, Fernández S, Agudelo B, Sánchez J. Años potenciales de vida perdidos por la población de Medellín 1994-1996. Medellín: Universidad de Antioquia; 1999.

6. Avalos Octavio, Rodríguez A. Mortalidad prematura por algunas causas de defunción seleccionadas, Cuba 1981. Rev Cubana Admin Salud 1987;13(1):103-107.

7. García C, López M. Medellín, mortalidad por causas en la década de los ochenta: una interpretación socio-demográfica. Invest Educ Enfermería 1995;13(1)

8. Colombia, Departamento Administrativo Nacional de Estadística. La mortalidad en Colombia en 1990. Boletin Estad 1991;462:209-211.

9. López M. Utilización de los datos de mortalidad para la planeación de los servicios de salud: en el caso de México. Rev Salud Publica Mexico 1985;27(2):124-138.

10. García C. Causas de muerte como indicadores indirectos de necesidades en salud: una interpretación socio-demográfica. Los casos de la Frontera Norte y de Morelos, 1980-1990. México, D.F.: Centro Regional de Investigaciones Multidisciplinarias (CRIM-UNAM); 1987.

11. Murray CJL, López AD. Global and regional cause of death patterns in 1990. Bull World Health Organ 1994;72(3):447-480.

12. Escobar ML, Gallardo HM, Giraldo GP, Londoño JL, Rodríguez J. La carga de la enferme- dad en Colombia. Santa Fe de Bogotá: Ministerio de Salud; 1994.

13. España, Dirección Xeral de Saúde Pública. Mortalidade evitable en Galicia 1976-1992: unha aproximación a través da diferencia da esperanza de vida. Documentos Tecnicos Saude Publica 1997; serie B(11):1-69.

14. Colombia, Departamento Administrativo Nacional de Estadística, Ministerio de Salud. Manual de principios y procedimientos: sistema de registro civil y estadísticas vitales (versión actualizada). Santa Fe de Bogotá DANE; 1997.

15. Grisales H, Agudelo B, Londoño JL. Estructura de la mortalidad según cinco primeras causas y su modelación temporal, Medellín 1987-1996. Revista Facultad Nacional de Salud Pública (Universidad de Antioquia) 1998; 16(4).

16. Murria CJL. Cuantificación de la carga de la enfermedad: la base técnica del cálculo de los años de vida ajustados en función de la discapacidad. Bol Oficina Sanit Panam 1995; 118(3):221-242. 
17. Londoño JL. La carga de la enfermedad: un nuevo indicador en el campo de la salud pública. Rev Facultad Nacional Salud Publica; 1996.

18. Escobar ML, Gallardo HM, Giraldo GP, Londoño JL, Rodríguez J. La carga de la enfermedad en Colombia. Santa Fe de Bogotá; 1994.

19. Rodríguez J, Gallardo M. Mortalidad y años de vida ajustados por discapacidad como medidas de la carga de enfermedad, Colombia 1985-1995. Santa Fe de Bogotá: Ministerio de Salud; 1999.

20. Colombia, Ministerio de Salud. La carga de la enfermedad en Colombia. Santa Fe de Bogotá: MS; 1994.

21. Banco Mundial. Informe sobre el desarrollo mundial, 1997. El estado en un mundo en transformación. Indicadores seleccionados del desarrollo mundial. Washington, D.C.: BM; 1998.

22. Bobadilla JR, Frenk J, Lozano R. Frejka T, Stern C. The epidemiologic transition and health priorities. En: Jamilson DT, Mosly WH, Measham
A, Bobadilla JL, eds. Disease control priorities in developing countries. Washington, D.C.: Oxford University Press; 1993. Pp. 52-53.

23. Rodríguez J, Gallardo H. La transición epidemiológica en Colombia, 1985-1995. En Ministerio de Salud, Instituto Nacional de Salud. Informe quincenal epidemiológico nacional, 4(14); 1999.

24. Yunes J. Mortalidad por causas violentas en la región de las Américas. Bol Oficina Sanit Panam 1993;114(4):302-315.

25. Grisales H, Fernández S, Cadena E. Años de vida saludable perdidos por la población de Medellín. Rev Facultad Nacional Salud Publica 1999;17(1):63-93.

26. Ruiz R M, Blanes Ll A, Viciana F F. Mortality among young people and its impact on life expectancy in Andalucía during 1980-1982. Rev Española Salud Publica 1997;71(2):139-148.

27. Notzon FC, Komarov YM, Ermakov SP, Sempos CT, Marks JS, Sempos EV. Causes of declining life expectancy in Russia. JAMA 1998; 279(10):793-800.
28. Yanagishita M, Guralnik JM. Changing mortality patterns that led life expectancy in Japan to surpass Sweden's: 1972-1982. Demography 1988;25 (4):611-624.

29. Loaiza I. Análisis de la mortalidad: apertura al potencial de intervención de causas evitables. Bol Epidemiol Antioquia 1993;18(1):6163.

30. García C. Mortalidad, crisis económica y costo de vida: análisis de coyunturas sociodemográficas. Medellín-Colombia, 1917-1985. Lecturas de Economía 1993;38:119-146.

Manuscrito recibido el 27 de abril de 2001. Aceptado para publicación, tras revisión, el 15 de julio de 2002.

ABSTRACT Objectives. To examine changes in life expectancy in Medellin, Colombia, between 1989-1991 y 1994-1996, in connection with four large groups of causes of death commonly employed in studies on the burden of disease: group 1, communicable disea-

Change in life expectancy in connection with three large groups of causes of death in Medellín, Colombia, between 1989-1991 and 1994-1996 ses, perinatal and maternal health problems, and nutritional deficits; group 2, noncommunicable diseases; group 3, wounds; group 4, ill-defined causes. The latter were excluded from the analysis because of their ambiguity.

Methods. The calculations were made according to the method described by J. H. Pollard in 1986, with the aid of Microsoft Excel, by using the recommended formulas. The computations were double checked with EPIDAT (version 3.0, unofficial).

Results. Between 1989-1991 y 1994-1996, a total gain of 1,93 years in life expectancy was seen in Medellin, with a rise from 62,13 to 64,06 years. The gain was greater in men than in women (2,42 vs. 1,09 years, respectively). The increase noted among females was greatest in the extreme age groups (girls 1 to 4 and women over 54 years of age); in men, it was highest in the middle years (between the ages of 25 and 44). In both sexes, the greatest percentage loss in life expectancy was seen in persons 15 to 19 years of age ( $23 \%$ in men and $4 \%$ in women, roughly).

In the group comprising communicable diseases, perinatal and maternal health problems, and nutritional deficits, a loss in life expectancy was seen in men $(0,04$ years), whereas in the group of non-communicable diseases there was a gain in life expectancy in both sexes (0,60 years among men and 0,55 years among women). The greater gain in life expectancy among men was linked to a reduction in mortality from wounds (1,98 years).

Conclusions. If one compares the results obtained in Medellin at the end of the study period with life expectancy at birth in Colombia in 1995, which was 70 years, it is obvious that life expectancy in Medellin is still lagging behind, even though it has risen progressively over the years. This is in keeping with the epidemiological transition the city has experienced, which has been linked with a rise in mortality from degenerative and cardiovascular diseases, chronic respiratory ailments, and diabetes, as well as from diseases resulting from human activity. Nevertheless, this epidemiological transition has been slow in Medellin when compared to Colombia as a whole due to high rates of death from infectious and parasitic diseases, which are more characteristic of the transition in its earlier stages. Despite the fact that mortality from wounds has decreased in Medellin, particularly among young males, it may be worthwhile to reassess the effectiveness of interventions undertaken in recent years to promote peaceful coexistence and tolerance in the community. 